\title{
A DETERMINATION OF THE CORRECTION FOR THE OPEN END OF A CYLINDRICAL RESONATOR.
}

By W. M. BоEнM.

$\mathrm{D}^{2}$ URING the past decade, several methods for measuring the intensity of sound, have been developed. Among the applications of these methods, that of the determination of the correction which must be added to the length of a cylindrical resonator, was considered worthy of re-determination.

The values of the correction which are generally quoted are those of Blaikley. ${ }^{1}$ His values give an average of $(.576) R(R$ being the radius of the resonator) for resonators having a flange of small width. For resonators having a flange of infinite width at the open end, Rayleigh ${ }^{2}$ has determined, by theoretical methods, that the correction is about (.82) $R$. For a flange of "zero" width, there is at present no theoretical determination.

In outline, the method used in this investigation was as follows: The source of sound was held near the open end of a cylindrical resonator the length of which was adjusted until the point of maximum resonance was found by one of the methods for measuring intensity of sound. In this way the position of one or more nodes was found. Wave-lengths and corrections were determined from the positions of these nodes. Observations were taken as rapidly as possible to avoid changes of humidity and temperature.

Resonators.-The resonators were made of drawn steel tubes, manufactured by the Seamless Tube Co., of Pittsburg. The thickness of wall was about $6.5 \mathrm{~mm}$. except a distance of $50 \mathrm{~mm}$. at the top which has turned to a thickness of 3.I mm. Heavy face-plates of cast iron were finished to fit the top closely. At the lower part, the tubes were mounted firmly with set-screws having

${ }^{1} D$. J. Blaikley, Experiments for Determining the Correction to be Added to the Length of a Cylindrical Resonant Tube, Phil. Mag. (V.), Vol. VII., p. 339, 1879.

${ }^{2}$ Lord Rayleigh, Theory of Sound, Vol. II., p. 487. 
conical points. The base $C$, Fig. I, was finished to fit the four resonators and to serve as a guide for the piston $\operatorname{rod} G$. Into the lower part of the base there was screwed a heavy round rod $H$. It served two purposes: upon it was engraved the scale by means of which measurements were made and the table $J$ could be fastened at any point to support the rack and pinion apparatus $K$. A clamp attached to $K$ served as a lever to make find adjustments. of the position of the piston. Readings were taken with a vernier having a least count of $.005 \mathrm{~cm}$. attached to the piston-rod at $I$. The piston $E$ was turned to a diameter slightly smaller than the inside diameter of the tube. In order to produce an air-tight fit, a leather washer having the diameter about $0.5 \mathrm{~mm}$. larger than the tube was clamped to the upper side by means of a plate $F$. It was then forced into the tube. The excess of leather was thus cut off by the sharp inner edge of the tube and a very closely fitting piston was produced. All parts of the resonator were made massive to make the surface of the metal as nearly as possible a surface of zero kinetic energy.

Source of Sound.-The attempt to obtain a source which would produce a sound of constant amplitude failed. Heavy tuningforks driven by electro-magnets could not be manipulated and smaller ones driven by the larger were not sufficiently constant. Organ pipes proved to be still more irregular. The most

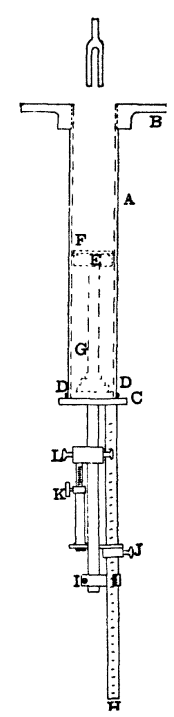

Fig. 1. reliable source was found to be a tuning fork which vibrates freely with a gradually decreasing amplitude. In making observations through the telescope due allowance must be made for the gradual but definite change in amplitude.

Position of Node.-To determine the position of a node accurately it was necessary to use the method of measuring sound with a vibration-galvanometer. ${ }^{1}$ The transmitter and resonator of this apparatus were placed a distance of about two meters from the resonator and either above or below the plane of the flanges. They were separated from the floor by cushions to prevent vibra-

${ }^{1}$ W. M. Boehm, A Method of Measuring Intensity of Sound, PHys. REv. 
tions from other sources from reaching the transmitter. The fork was struck with a hammer made of rubber and the piston moved to a position near the node. Finer adjustments were made with the rack-and-pinion apparatus while observing the vibrations of the galvanometer. Before the vibrations become inaudible, the piston can several times be gradually shifted through the point of maximum resonance. The forks used had frequencies of about $384,768, \mathbf{I}, 536$ half-vibrations per sec.

Maxima of Intensity.-When the fork is very near the opening of the resonator, the intensity increases very sharply as the piston is moved through the proper position. See curve no. I, Fig. 2. If

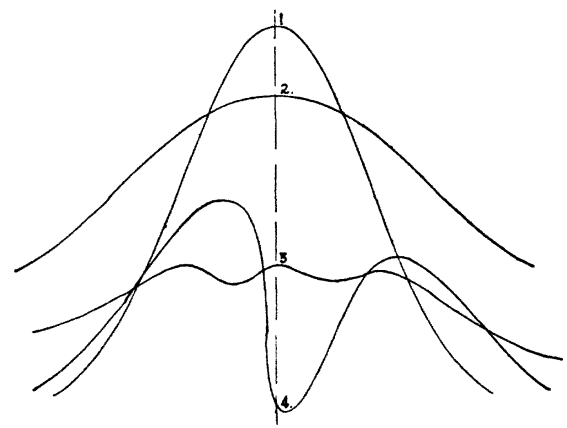

Fig. 2. the fork is farther away the increase is not so sharp, no. 2, Fig. 2. If the fork is removed still farther it is possible to find a position at which there appear to be three maxima though not very marked. The curve no. 3, Fig. 2, is exaggerated to show this peculiarity. The third maximum is less intense than the first. In curve no. 6, Fig. 4, three of these maxima are shown for a distance of about $8.4 \mathrm{~cm}$. between resonator and fork. On moving the fork still farther it is found that two maxima of unequal magnitude can be obtained (see no. 4, Fig. 2). The first and more intense maximum seems to be displaced from the point where maximum resonance would be expected. The curves, roughly shown in Fig. 2, are not produced by the same amplitude of vibration, but merely represent how the intensity varies as we pass through a maximum. The middle line is also shifted to coincide for the four curves.

These results point to a caution. If the apparatus which is to detect the intensity of sound is outside the resonator, a maximum of intensity may not indicate "unison" between the resonator and the wave-length or frequency produced outside. This is especially true for distances greater than $8 \mathrm{~cm}$. between the resonator and fork. 
Distance of Fork and Position of Node.-The fork having a frequency of about 768 half-vibrations per second was placed with its prongs pointing downward parallel to the axis of the resonator. The point of maximum resonance was obtained with a fork at different distances from the resonator. The observations are shown in curve no. I, Fig. 3. It can be seen that the position of the node
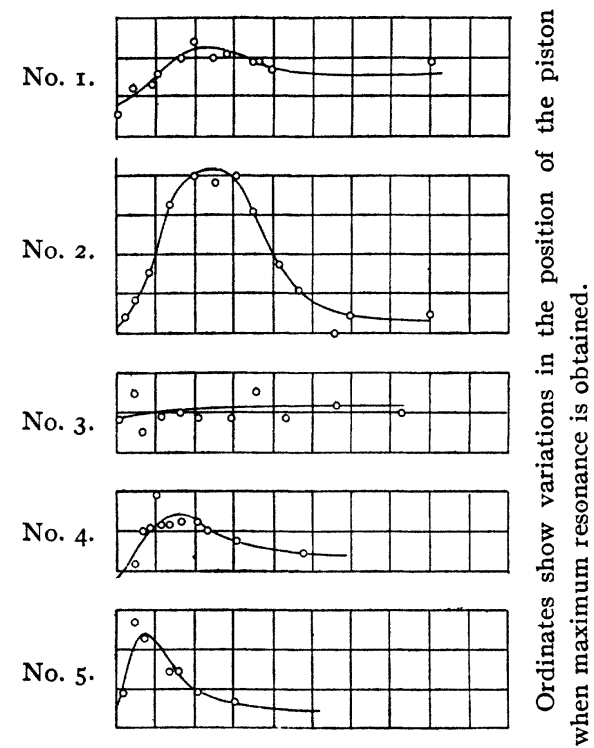

Fig. 3.

Absciss $æ$ are proportional to distance from mouth of the resonator to the fork.

and therefore the length of the resonator varies as the distance to the fork is altered. First the resonator becomes longer until the fork is about $2.5 \mathrm{~cm}$. from the opening, then it becomes shorter until it becomes apparently constant.

To ascertain this effect on a node nearer the end of the tube, the fork having a pitch an octave higher was selected. The curve obtained is shown as no. 2, Fig. 3. Using the same fork but the third node instead of the first, gave a curve which is not so marked, no. 3, Fig. 3. Resonance being very feeble, the observations do not agree so well as those for the upper node.

To ascertain the effect of width of flange upon the character of 
the curve, another pipe was selected and the fork of 768 vibrations used. The curve no. 4, Fig. 3, shows the variation in position of the node when the width of flange was $0.3 \mathrm{I} \mathrm{cm}$. and no. 5 the curve when the width of flange was $13.34 \mathrm{~cm}$. If these indicate anything conclusive it may be that, in the case of the larger flange, the least correction, i. e., the maximum of the curve is nearer the origin or when the fork is nearer the resonator.

All curves of the upper node show a decided maximum and make it evident that an average of the observations cannot be taken as the true position of the node. A more correct value would be that obtained when the fork is a great distance from the tube. This distance would probably be about 15 to $20 \mathrm{~cm}$. in the case of the fork generally used in these experiments. Since the observations become uncertain at such great distance it is necessary to assume

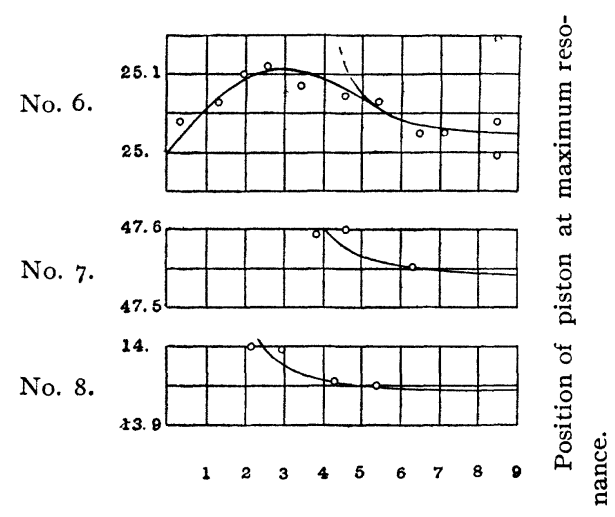

Fig. 4.

Distance from fork to resonator.

that the curve is a straight line parallel to the $X$-axis in this neighborhood. If, then, we select some curve which approaches an asymptote we can, by comparing it with a rather complete curve, ascertain what parts of the two curves coincide and take observations along these parts. The template at hand was that of an equilateral hyperbola which, in no. 6, Fig. 4, is shown drawn farther toward the origin to indicate where it no longer conforms to the outline of the observations. To obtain values for calculations we may take the ordinates when the fork is 15 or $20 \mathrm{~cm}$. from the 
resonator. In case of nodes farther than $50 \mathrm{~cm}$. from the end of the tube, the observations are taken at such a distance that averages of observations can be taken without the use of the curves.

Effect of Wave-length.-To ascertain the effect of wave-length on correction, three forks were selected; the one generally used having 768 half-vibrations per second, one an octave higher and the third an octave lower. On comparison they were found to differ by an octave to within one tenth vibration per second. Allowing the greatest difference as one tenth vibration an error, in the correction, of $.05 \mathrm{~cm}$. could enter into the final result. Curves obtained with these forks are shown in Fig. 4.

\begin{tabular}{c|c|c|c|c}
\hline Curve. & Fork. & Upper Node. & Correction. & \\
\cline { 2 - 5 } No. 7 & 384 & 47.545 & 2.052 & 44.827 \\
No. 6 & 768 & 25.035 & 2.150 & 22.413 \\
No. 8 & 1.536 & 13.945 & 2.032 & 11.207 \\
\hline
\end{tabular}

Position of lower node, $69.860 \mathrm{~cm}$. for fork 768 .

Top of resonator, $4.770 \mathrm{~cm}$.

Temperature, $23^{\circ} .55 \mathrm{C}$.

It will be seen from these observations that the correction for the fork of lowest pitch and for that of highest pitch are nearly alike but the intermediate pitch gives a value a little higher. This may, however, be due to errors in comparison of forks and in observation. Taken together the values point to the conclusion that the correction is independent of wave-length.

Wave-lengths.-Each of the four resonators was provided with four flanges and its own thickness as a fifth flange. The positions of the upper nodes were determined from the curves for each flange (see Figs. 5, 6, 7 and 8). The wave-length was assumed to be twice the distance between the first and second node. Wave-length was determined once for each series of observations which were taken rapidly to avoid changes in humidity. Changes due to temperature were calculated.

Determination of Correction.-From the values of quarter wavelengths as corrected for temperature, and the positions of the nodes as obtained from the ordinate when the fork may be considered far 
from the resonator, the correction can be determined by the use of the formula:

$$
x=\lambda / 4-\left(L_{1}-L_{2}\right) .
$$

Wave-lengths as Determined from the First and Second Nodes without a Face-plate on the Resonator.

\begin{tabular}{c|c|c|c|c}
\hline \hline Resonator. & $\begin{array}{c}\text { Upper Node } \\
\text { (from Curves). }\end{array}$ & $\begin{array}{c}\text { Lower Node } \\
\text { (from Observ.). }\end{array}$ & $\begin{array}{c}\text { Temperature } \\
\text { (Observed) }\end{array}$ & $\lambda$ \\
\cline { 2 - 5 } 1 & 23.440 & 68.290 & 22.7 & 22.425 \\
2 & 24.085 & 69.090 & 24.6 & 22.503 \\
3 & 25.065 & 69.950 & 23.5 & 22.442 \\
4 & 24.890 & 69.650 & 21.3 & 22.380 \\
\hline
\end{tabular}
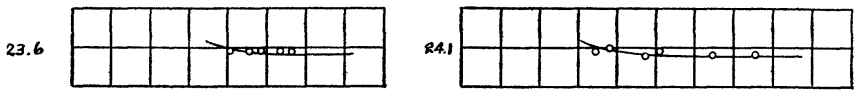

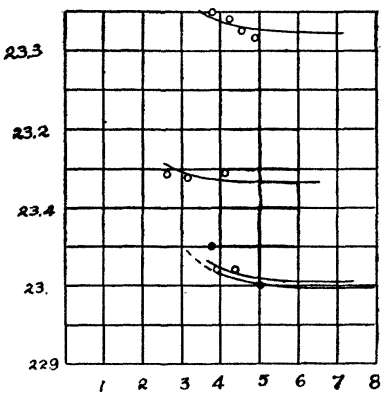

Fig. 5.
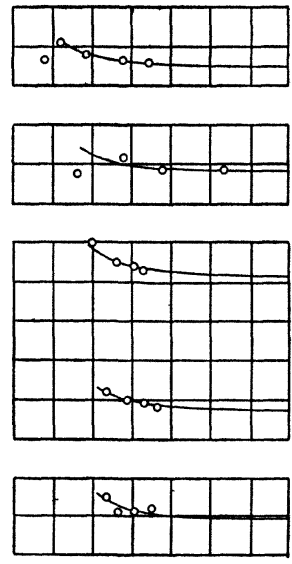

Fig. 7.

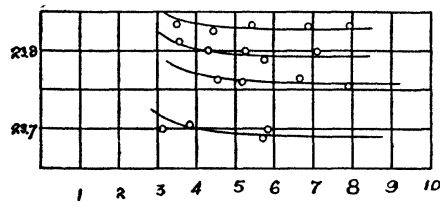

Fig. 6.
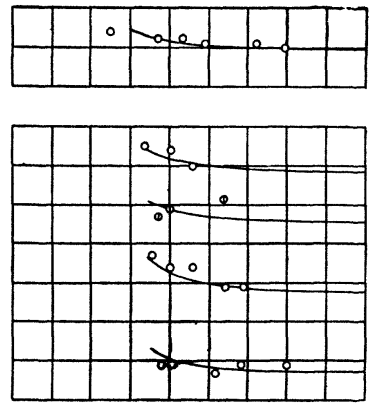

Fig. 8. 
$x=$ correction due to open end.

$\lambda=$ wave-length.

$L_{1}=$ position of the upper node.

$L_{2}=$ position when the top of the piston is in the same plane as the top of the resonator.

Plotting the relation between width of flange and correction, we obtain the average curve shown in Fig. 9. As may be expected, each curve indicates a tendency to approach a constant value as the width of flange is increased. This enables us to estimate the correction for an infinite flange or, extending the curve in the opposite direction, to determine the correction for a flange of "zero" width. The following table shows a series of the observations.

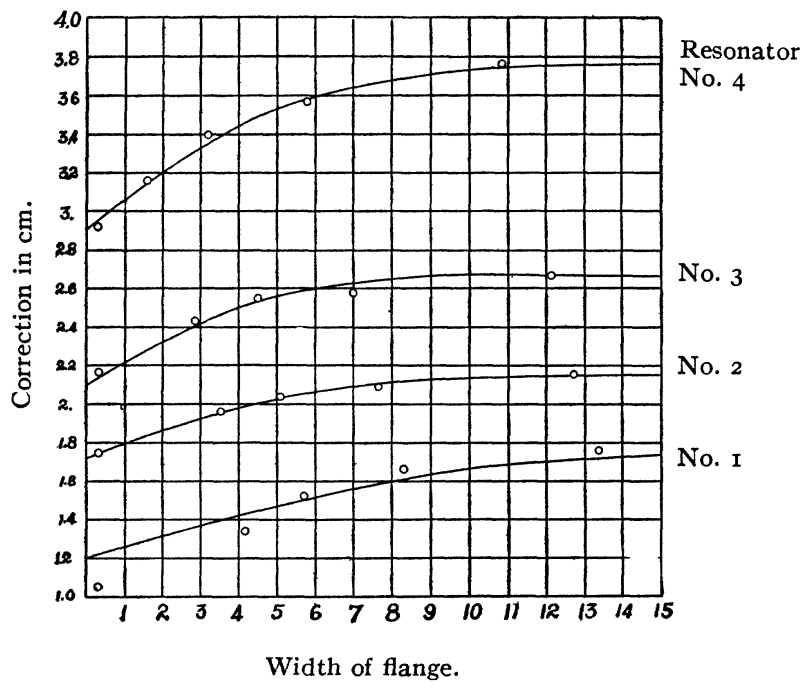

Fig. 9.

Average Correction.-From the above results we see that for a "zero" flange we have the average correction $(.656) R$ which is 15 per cent. above the values obtained by Blaikley. For an infinite width of flange the average is $(.87 \mathrm{I}) R$ which is about 6.5 per cent. above the probable value given by Rayleigh. To test whether these high results were due to the presence of the ceiling which was only $90 \mathrm{~cm}$. above the top of the resonator, the whole apparatus was removed to a 


\begin{tabular}{|c|c|c|c|c|c|c|c|c|c|c|}
\hline \multirow{2}{*}{$\begin{array}{l}\text { Reso- } \\
\text { nator. }\end{array}$} & \multicolumn{3}{|c|}{ From Curve. } & \multicolumn{3}{|c|}{ No. o. } & \multicolumn{3}{|c|}{ No. I. } & \multirow{2}{*}{$\begin{array}{c}\text { Top } \\
\text { at }\end{array}$} \\
\hline & Asym. & Temp. & $\lambda / 4$ & Asym. & Temp. & $\lambda / 4$ & Asym. & Temp. & $\lambda / 4$ & \\
\hline 1 & 23.44 & 22.7 & 22.425 & 23.59 & 22.7 & 22.425 & 23.32 & 22.9 & 22.429 & \\
\hline 2 & 24.085 & 24.6 & 22.503 & 24.085 & 24.6 & 22.503 & 23.880 & 24.75 & 22.506 & \\
\hline 3 & 25.065 & 23.5 & 22.442 & 25.045 & 23.5 & 22.442 & 24.785 & 23.55 & 22.443 & \\
\hline \multirow[t]{2}{*}{4} & 24.89 & 21.3 & 22.380 & 24.925 & 21.3 & 22.380 & 24.685 & 21.3 & 22.380 & \\
\hline & \multicolumn{2}{|c|}{ No. 2.} & & \multicolumn{2}{|c|}{ No. 3.} & & \multicolumn{2}{|c|}{ No. 4.} & & $\mathrm{~cm}$. \\
\hline 1 & 23.135 & 22.8 & 22.427 & 23.005 & 22.8 & 22.427 & 22.995 & 22.9 & 22.429 & 2.230 \\
\hline 2 & 23.795 & 24.8 & 22.507 & 23.755 & 24.9 & 22.509 & 23.685 & 24.95 & 22.510 & 3.335 \\
\hline 3 & 24.725 & 23.6 & 22.444 & 24.635 & 23.65 & 22.445 & 24.540 & 23.6 & 22.444 & 4.770 \\
\hline \multirow[t]{3}{*}{4} & 24.445 & 21.4 & 22.382 & 24.275 & 21.4 & 22.382 & 24.090 & 21.5 & 22.384 & 5.465 \\
\hline & \multirow{2}{*}{\multicolumn{2}{|c|}{ From Curve. }} & \multicolumn{2}{|c|}{ No. o. } & \multicolumn{2}{|c|}{ No. $\mathbf{r}$} & \multicolumn{2}{|c|}{ No. 2.} & & \\
\hline & & & $\begin{array}{l}\text { Dist. to } \\
\text { Top. }\end{array}$ & Corr. & $\begin{array}{l}\text { Dist. to } \\
\text { Top. }\end{array}$ & Corr. & $\begin{array}{c}\text { Dist. to } \\
\text { Top. }\end{array}$ & Corr. & & \\
\hline 1 & 21.21 & 1.215 & 21.360 & 1.065 & \begin{tabular}{|l|l}
5 & 21.090
\end{tabular} & 1.339 & 20.905 & 1.522 & & \\
\hline 2 & 20.750 & 1.753 & 20.750 & 1.753 & $\begin{array}{ll}320.545 \\
\end{array}$ & 1.961 & 20.460 & 2.047 & & \\
\hline 3 & 20.295 & 2.147 & 20.275 & 2.167 & 20.015 & 2.428 & 19.955 & 2.552 & & \\
\hline \multirow[t]{2}{*}{4} & 19.425 & 2.955 & 19.460 & 2.920 & 19.220 & 3.16 & 18.980 & $|3.402|$ & & \\
\hline & & & \multicolumn{2}{|c|}{ No. 3 . } & \multicolumn{2}{|c|}{ No. 4.} & & & & \\
\hline 1 & & & 20.775 & 1.652 & 20.765 & 1.664 & \multirow{4}{*}{\multicolumn{4}{|c|}{ Correction in $\mathrm{cm}$. }} \\
\hline 2 & & & 20.420 & 2.089 & 20.350 & 2.16 & & & & \\
\hline 3 & & & 19.865 & 2.58 & 19.770 & 2.674 & & & & \\
\hline \multirow[t]{2}{*}{4} & & & 18.810 & 3.572 & $\mid 18.625$ & 3.759 & & & & \\
\hline & $\begin{array}{r}\text { Inside } \\
\text { Radius. }\end{array}$ & No. o. & No. 1. & No. 2. & No 3 . & No. 4 . & & & & \\
\hline 1 & 1.903 & .328 & 4.164 & 5.715 & 8.305 & 13.34 & \multirow{4}{*}{\multicolumn{3}{|c|}{ Width of flange. }} & \\
\hline 2 & 2.545 & .313 & 3.483 & 5.072 & 7.634 & 12.697 & & & & \\
\hline 3 & 3.181 & .310 & 2.848 & 4.437 & 6.998 & 12.062 & & & & \\
\hline 4 & 4.447 & .310 & 1.581 & 3.170 & $\mid 5.732$ & 10.795 & & & & \\
\hline
\end{tabular}

\begin{tabular}{c|c|c|c|c|c}
\hline \multirow{2}{*}{ Resonator. } & \multirow{2}{*}{ Radius Inside. } & \multicolumn{2}{|c|}{ "Zero" Flange. } & \multicolumn{2}{c}{ "Infinite" Flange. } \\
\cline { 3 - 6 } & & $x$ & $x / R$ & $x$ & $x / R$ \\
\hline 1 & 1.903 & 1.20 & .631 & 3.8 & .855 \\
2 & 2.545 & 1.73 & .680 & 2.7 & .849 \\
3 & 3.181 & 2.11 & .663 & 2.19 & .861 \\
4 & 4.447 & 2.90 & .652 & 1.73 & .919 \\
\hline
\end{tabular}

room in which the ceiling was about $600 \mathrm{~cm}$. above the resonator. The results obtained were practically the same except in the case of 
resonator no. I. For this they conformed more nearly to the outline of the curve as drawn in Fig. 9 than observation there shown.

Conclusions.- The length of a resonator responding most to the pitch of a fork depends upon the distance of the fork from the open end. ${ }^{1}$ This effect becomes more marked as the resonator becomes shorter and the fork is nearer the open end. A maximum of intensity outside the tube may not indicate that the resonator is nearest to unison with the pitch produced. The correction for a flange of "zero" width is about (.656) $R$ and for a flange of infinite width is about $(.87 \mathrm{I}) R$.

At this point I wish to thank my colleagues, at the Randal Morgan Laboratory, for suggestions and assistance. Especially do I wish to thank Prof. H. C. Richards for suggesting the subject, and Dr. R. H. Hough for suggestions and assistance in obtaining apparatus.

RANDAL MORGan LABORATORY,

University of Pennsylvania, May, Igro.

${ }^{1}$ R. H. M. Bosanquet, Notes on the Theory of Sound, Phil. Mag., Series 5, Vol. IV., p. 216,1877 . 